This is a postprint version of the following published document:

J. L. Pérez-Castellanos, D. S. Montero, C. Vázquez, J. Zahr-Viñuela, M. González. "Photo-thermo-mechanical behavior under quasi-static tensile conditions of a PMMA-core optical fiber". Strain 52 (2016) 1, pp. 3-13. Available in http://www.dx.doi.org/10.1111/str.12152

(C) 2015 Wiley 


\title{
Photo-Thermo-Mechanical Behaviour Under Quasi- Static Tensile Conditions of a PMMA-Core Optical Fibre
}

\author{
J. L. Pérez-Castellanos*, D. S. Montero ${ }^{\dagger}$, C. Vázquez ${ }^{\dagger}$, J. Zahr-Viñuela* and M. González \\ * Departamento de Mecánica de Medios Continuos y Teoría de Estructuras, Universidad Carlos III de Madrid, Leganés, Spain \\ E-mail: percaste@ing.uc3m.es \\ †Grupo de Displays y Aplicaciones Fotónicas del Departamento de Tecnología Electrónica, Universidad Carlos III de Madrid, Leganés, Spain \\ ‡Departamento de Ciencia e Ingeniería de Materiales e Ingeniería Química, Universidad Carlos III de Madrid, Leganés, Spain
}

\begin{abstract}
As the optical power transmitted by an optical fibre under tensile stress varies with strain, it can be used as a sensor for strain monitoring in structural elements. In the present work, quasi-static tensile tests of step index polymer optical fibres (POF) with simultaneous measurement of surface temperature and optical power are described. Young's modulus, yield stress and tensile strength are derived from experimental tests. Morphological characterization of the POF fibres using scanning electron microscope images and differential calorimetry technique is performed. The contributions of both elastic and plastic strain components to the variation of temperature and optical power loss are also estimated. The evolution of the POF mechanical properties as well as that of temperature and optical power loss is explained in terms of the progressive relative movement and alignment of the molecular chains in the direction of the applied load. Strain, temperature and optical power loss are then correlated.
\end{abstract}

KEY WORDS: field strain, mechanical behaviour, microstructural analysis, optical power loss, polymer optical fibre, temperature increase.

\section{Introduction}

Optical fibres include a transparent core material surrounded by a transparent cladding material with a lower index of refraction. Consequently, a ray of light can be propagated throughout the fibre core by means of the total internal reflection mechanism causing the fibre to act as a waveguide thus assuring the light guiding condition. Optical fibre sensors may use any light property to measure an external disturbance that can be detected by means of a photodetector. The optical sensing technology has several advantages compared to other sensing solutions as it exhibits a set of very attractive characteristics, including immunity to electromagnetic interference, small-size, great resistance to hostile environments that may comprise hazardous chemicals or inflammable atmospheres, sensor multiplexing as well as performing quasi-distributed sensing over a single fibre. Particularly, polymer optical fibres (POF) exhibit higher light coupling efficiency, leading to lower cost sensing schemes, and provide more flexibility compared to silicabased optical fibre solutions, the latter feature directly related to the elastic-plastic properties of the polymeric materials from which they are manufactured. PMMA-based POF fibres can be strained more than $40 \%$ because of their elastic-plastic properties while fully maintaining their light guiding properties. These benefits as well as the potential to use different optical techniques to measure strain such as optical power loss POF variations, central wavelength shifts on fibre Bragg gratings (FBG) manufactured in POF, or distributed sensing enable their use in structural health monitoring (SHM) applications, for instance applied to cracking detection or fatigue analysis [1]. If they are based on monitoring the intensity level of optical signals, standard referencing techniques can be used to avoid potential errors related to external power fluctuations that have already been developed for the case of addressing POF-based sensors [2, 3]. Most of the conventional sensors used in the above mentioned SHM applications are based on transmission of electric signals and have some limitations. These sensors are usually not small or durable enough to be surface mounted or embedded in a structure to measure interior properties. They are local (or point) sensors, which are restricted to measure only parameters at one location and cannot be easily multiplexed. The long lead lines also pose problems for large civil structures, which often span several or tens of kilometres. In some cases, the signals could not be discriminated from noise because of electrical or magnetic interference (EMI). In addition, various demodulation techniques are required for different sensors.

Quasi-static tests of self-made POF specimens with a mixture of monomers in the core and poly(methyl methacrylate) (PMMA) in the cladding have been performed, and a yield strain value of $6.1 \%$ has been 
measured [4]. Optical power loss measurements using POFs as mechanical sensors have already been discussed in [5] and a (normalized) sensitivity of $1.8 \times 10^{-5} / \mu \varepsilon$ is reported. POF fibre with $980-\mu \mathrm{m}$ core diameter, 1.492 core refractive index (RI) and a $20-\mu \mathrm{m}$ cladding of a fluorinated polymer $(\mathrm{RI}=1.405)$ is used. The yield stress and the Young's modulus of the PMMA-based core material are found to be $82 \mathrm{MPa}$ and $3.09 \mathrm{GPa}$, respectively. But there is no thermal analysis, although the mechanical properties of polymers generally show a high dependence on both temperature and strain rate. If the temperature approaches the glass transition temperature, a sharp decline in their mechanical properties appears; the material loses its strength and its resistance to deformation. A greater strain rate strengthens the material as shown in [6] where tensile tests of POF specimens at different strain rates are performed.

Work done by external forces during the deformation of a body is transformed into energy that is stored in the material. Only a fraction of this energy can be recovered if the solid is unloaded. The recoverable part of the energy manifests itself in two forms: as the elastic part of the strain tensor-with its associated elastic strain energyand as a temperature variation in a process that is thermodynamically reversible. The non-recoverable part manifests itself also in two forms: as the plastic part of the strain tensor-with its associated plastic work (cold work) which is, in turn, associated to some permanent changes related to the microstructure of the materialand also as a temperature increase (i.e. meaning heat generation). It is well established that the amount of heat generated during plastic straining is usually considered a constant fraction of the plastic work [7].

The surface temperature variation of a specimen during testing may be measured by infrared thermography techniques which require the use of radiometrically calibrated infrared cameras. Several authors have developed techniques for measuring the temperature increase that occurs in materials (primarily metal) under quasi-static deformation processes [8]. However, similar studies for polymers are less frequent.

Mechanical deformation of a fibre under tension consists of both axial elongation and simultaneous reduction on its cross-sectional diameter. If a light beam propagates through the deformed fibre its optical path is altered because of fibre elongation and RI changes. The optical power variation can be then correlated with the deformation suffered by the fibre, which is the basis of the use of optical fibres as intensity-based strain sensors.

In the present work, the relationship between the strain applied on a POF and the resulting variation in temperature and optical power loss is investigated by means of quasi-static tensile tests performed on PMMA- based POFs with simultaneous measurement of specimen surface tempe-rature and received optical power. From the tests, the main mechanical parameters of the fibre material are also obtained, such as Young's modulus, yield stress and tensile strength. Moreover, the characterization of the tested optical fibres using scanning electron microscope images (SEM) and differential calorimetry (DSC) techniques is also addressed.

In the following sections a comprehensive description of the experimental setup, materials and methods is provided. Finally the results are discussed.

\section{Experimental Techniques}

\section{Material and geometry of the specimens}

A standard 980- $\mu \mathrm{m}$ PMMA-based core-diameter step-index multimode POF fibre (Agilent HFBR-RUS500) is employed during the tensile tests. Its cladding performs a $10-\mu \mathrm{m}$ thickness concentric layer composed of a fluorinated polymer (typically fluoroalkylmethacrylate). Figure 1-A depicts a scheme of the optical fibre physical structure used for the tests.

The calibration length used for the tensile tests of POFs specimens-defined as the distance between both points of load application-is of $100 \mathrm{~mm}$. However, total length of fibre specimens is about $1000 \mathrm{~mm}$, necessary to reach both transmitter and receiver optical circuits. At the points of load application, the fibre is glued to a pair of aluminium plates of different size. In this way, the jaws of the testing machine applied pressure on the longer plate, while the shorter plate assures a given thickness of the adhesive used to glue the fibre (Figure 1-B).

\section{Testing procedure}

\section{Tensile tests}

Quasi-static tensile tests are performed by controlling the displacement (constant relative velocity prescribed between the points of load application) using an Instron Universal Testing Machine, Model: 8516/8802, with a maximum load capacity of $100 / 250 \mathrm{kN}$. To determine the influence of the strain rate in quasi-static conditions several tests are made at different load velocities. The defining parameter for each tensile test is the value of the prescribed load velocity.

Provided the fact that the fibre core diameter is much larger than the cladding thickness, the system formed by core and cladding can be considered as a single, homogeneous material, making it suitable to calculate the true values of stress and strain. 


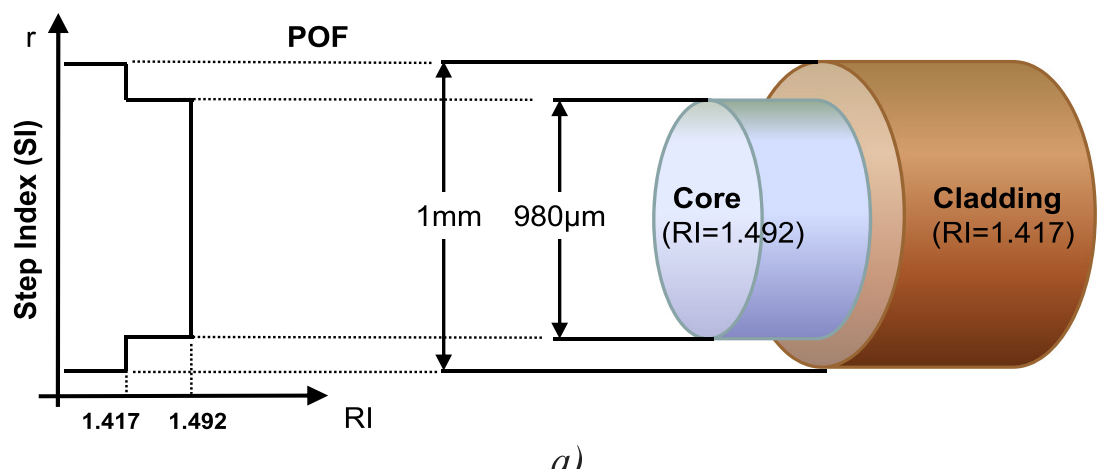

a)

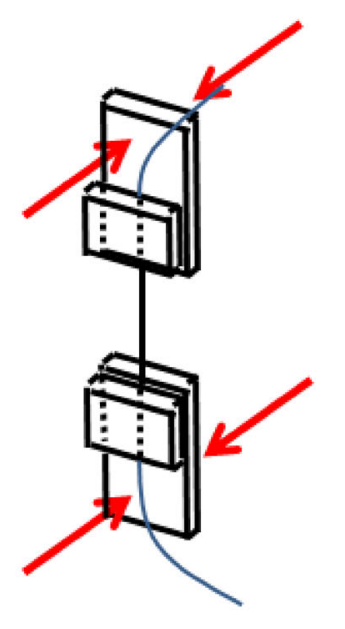

b)

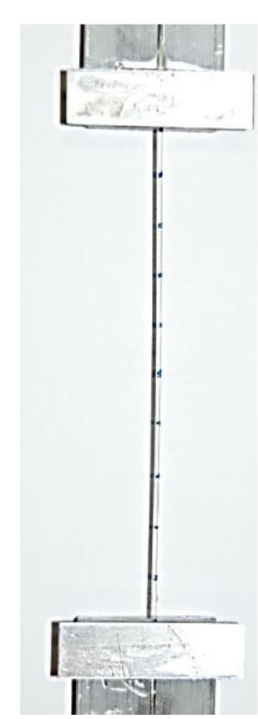

c)

Figure I: (a) POF fibre geometry, (b) specimen mounting scheme, (c) specimen showing the dot markings

As a direct result of the test, the time evolution of applied force, $\mathrm{F}=\mathrm{F}(\mathrm{t})$, and specimen elongation, $\Delta \mathrm{L}=\Delta \mathrm{L}(\mathrm{t})$, are recorded. From these, the time evolutions of nominal and true strain are calculated as in Equations (1.a) and (1.b), where $\mathrm{L}_{0}$ is the initial gauge length. Nominal and true stresses are calculated according to classical expressions of Equations 1.c and 1.d, where $A_{0}$ is the initial area of the transversal section of the fibre, and where fibre incompressibility is assumed for the calculation of the true stress. The $\sigma-\varepsilon$ curve is built by time-matching the results of Equations (1.c) and (1.d).

$$
\begin{aligned}
& e_{N}(t)=\Delta L(t) / L_{0} ; \\
& \varepsilon(t)=\ln \left(1+e_{N}(t)\right) \\
& S_{N}(t)=F(t) / A_{0} ; \\
& \sigma=S_{N}(t)\left\{1+e_{N}(t)\right\}
\end{aligned}
$$

From $\sigma-\varepsilon$ curve, Young's modulus, $E$, yield stress, $\sigma_{\mathrm{y}}$, tensile strength, $S_{m}$, and ultimate strain, $\varepsilon_{R}$, can be calculated. The elastic and plastic strain components are determined using the additive decomposition of the total strain, as seen in Equation (2).

$$
\varepsilon^{e}=\frac{\sigma}{E} \quad \varepsilon^{p}=\varepsilon-\frac{\sigma}{E}
$$

\section{Temperature measurements}

During the mechanical tests, the temperature variation associated with material strain is measured on the specimen surface. For measuring the increase in temperature of the calibration length of the fibre during its deformation process, an uncooled infrared camera (IRC) of high sensitivity, FLIR SC600, is used. For temperature calculations, a PMMA emissivity value of 0.86 is considered [9]

For minimizing undesirable temperature recordings of the specimen surface because of variations in the surrounding environment, IRC camera is protected during the tests with 
a chamber of insulating material leaving a window for data collection.

If the strain distribution is considered to be uniform inside the fibre, the heat generation and its associated temperature increase should also be uniform inside the fibre, preventing heat conduction in both axial and radial directions.

The duration of the test cannot be, rigorously, considered as short enough as to guarantee true adiabatic conditions. The surface specimen temperature could be affected at first by natural convection with the surrounding air inside the insulating chamber. Even if the insulating chamber avoids as much as possible heat exchanges with the surrounding environment, the temperature distribution inside the fibre could also be non-uniform. Nevertheless, the temperature increase measured at the specimen surface is relatively small (as shown below) which suggests negligible effects of natural convection.

Because of these considerations, the temperature measurements taken at the specimen surface are considered to be a good representation of the internal temperature.

\section{Optical power measurements}

Light is transmitted through the specimen during the mechanical tests taking simultaneous measurements of the optical power losses. The signal conditioning circuit at the reception stage includes both amplifying and filtering stages.

To avoid undesirable variations in the measured optical power because of short-term optical source fluctuations or external perturbations that could distort the analysis of the experimental results, the received optical power of a second unloaded POF (reference channel) is additionally monitored. Such an optical scheme provides selfreferencing properties on the light intensity measurements in which the reference channel bypasses the specimen under tests and reaches the reception stage. Optical power measurements of both the POF specimen and the reference channel are then correlated.

On the other hand, the time scale of both the temperature and received optical power measurements are correlated with the time scale of the mechanical test.

\section{Morphological characterization}

Fracture surfaces are observed by using the scanning electron microscopy (SEM) technique. SEM images are obtained with a Philips XL 30 microscope equipped with an EDAX microanalysis probe. Thermal transitions are studied by differential scanning calorimetry (DSC) on a Mettler Toledo 822 at $20^{\circ} \mathrm{C} / \mathrm{min}$.

\section{Results and Discussions}

\section{Mechanical behaviour}

All tests are carried out at room temperature, from 20 to $21^{\circ}$ $\mathrm{C}$, and until fibre breakage.

To take into account the possible scatter of results, a set of three measurements are made for each test condition (defined by the value of the load application velocity). The experimental results showed statistically irrelevant deviations in all cases.

\section{Influence of coating presence}

Initially, tests were carried out using jacketed POF specimens. However, numerous practical problems related not only with the affixing of the specimen in the testing machine but also with difficulties in measuring the surface temperature and the true values of strains and stresses, lead to remove the fibre jacket from the specimens before the tensile tests.

The contribution of the jacket layer to the fibre strain dependency is verified by conducting a reduced set of simplified tests with jacketed specimens at the same test conditions (load velocity) with respect to unjacketed counterparts. Comparison of force versus elongation experimental results in both specimens demonstrated that the influence of the jacket layer on the fibre stiffness can be considered as negligible.

\section{Observations regarding strain localization and fracture}

In order to observe if strain localization effects take place, specific tests are performed with specimens marked every $10 \mathrm{~mm}$ with ink dots along the calibration length (see Figure 1-C). Four tests are performed: (1) stopped after the yield point, (2) stopped at the strain value corresponding to the local minimum of the stress-strain curve, (3) stopped near to the fracture point and (4) until specimen breakage.

After each test, the spaces between the dots printed in the calibration length are measured. For tests (1) and (2), it is showed that the strain field is essentially uniform across the specimen. For test (3), the elongation of the specimen is almost uniform, without unambiguous evidence of necking or strain localization. For test (4), necking is still not clearly observable. If severe strain localization occurred, they remained hidden by the fracture zone.

From all tests, it is also found that the fibre diameter decreased by $15-20 \%$, being this reduction also uniform.

\section{True stress versus true strain curve}

Figure 2 depicts an illustrative true stress versus true strain $(\sigma-\varepsilon)$ curve obtained in a test at a load velocity of 


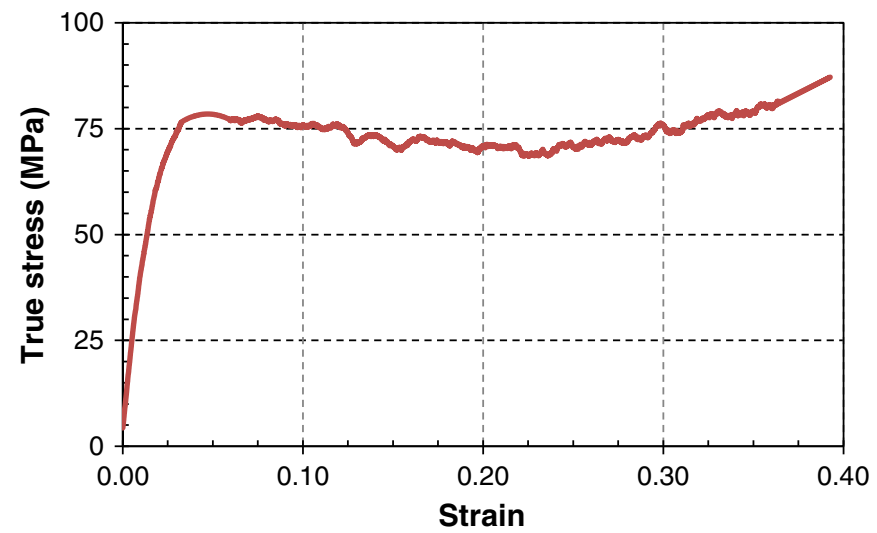

Figure 2: Measured curve $\sigma-\varepsilon$ obtained in a $0.0005 \mathrm{~s}^{-1}$ strain rate test

$4 \mathrm{~mm} / \mathrm{min}$ corresponding to a strain rate of $0.0005 \mathrm{~s}^{-1}$. This figure is similar to those obtained by several authors [10].

Before the material reaches the yield stress, corresponding to strains around 0.04-0.05, the intermolecular forces that bind the molecular chains are still able to balance the external forces. Therefore, if the load is removed the chains, in fact, recover their initial position which is macroscopically manifested as a reversible (elastic) deformation.

Exceeding the yield stress (minimum value of stress above which plastic strain occur) implies that the intermolecular forces cannot balance the applied stress. Relative displacements between chains occur until new stable positions are reached, which is macroscopically manifested as an irreversible (plastic) deformation. During this phase the permanent deformations can greatly increase with small stress variations. The material is thus able to absorb a large amount of energy by deformation. The relative shift in the molecular chains to stable positions is accompanied by their progressive orientation in the direction of the applied load. At $\varepsilon \approx 0.10$ an inflexion point on the $\sigma-\varepsilon$ curve is observed. This fact suggests that this strain value could be established as a boundary between a deformation state in which the molecular chains mostly reach stable relative positions (permanent deformations occurring in the material) and a deformation state in which the chains mostly begin to orient themselves in the same direction as to the applied load. When most of the molecular chains are already oriented a progressive macroscopic hardening of the polymer occurs, which is reflected in the positive slope of the last region (hardening region) of the $\sigma-\varepsilon$ curve.

From $\varepsilon \approx 0.04-0.05$ until $\varepsilon \approx 0.2$ a load drop is observed (negative slope of the $\sigma-\varepsilon$ curve). This load drop is sometimes attributed in the literature [11] to a thermal softening effect, which is because of an adiabatic heat generation associated to the plastic deformation of the material. However, according to the experimental temperature measurements reported below, temperature increase in this strain range is not sufficient to explain the load drop in terms of a thermal softening effect of the material. Sometimes the load drop is attributed to the appearance of a localized instability (necking). However, the observations carried out in the present work on the deformation processes show that the elongation is essentially uniform. More than an observable localized necking, it can be considered that during this strain range, a process of material softening-perhaps because of some form of internal damage of the material-took place throughout the entire specimen length.

\section{Microstructural analysis of deformed fibre}

\section{Morphological characterization by scanning electron microscopy (SEM)}

Scanning electron microscopy (SEM) is used to characterize the morphology of the fibre and the influence of the tensile stress on it. Samples are taken close and far from the fracture area and sections perpendicular and parallel to the strain direction are inspected. Some illustrative examples of the obtained images are depicted in Figure 3.

In Figure 3-A, a SEM image of the long section of the fibre is shown. Two different regions are observed: a thin layer (roughly $2-\mu \mathrm{m}$ thickness) in the outer part of the section and a smooth continuous and homogeneous region corresponding to the inner part of the fibre. X-ray

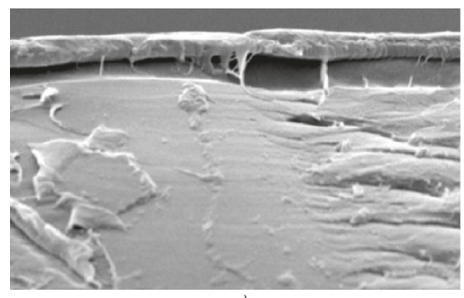

a)

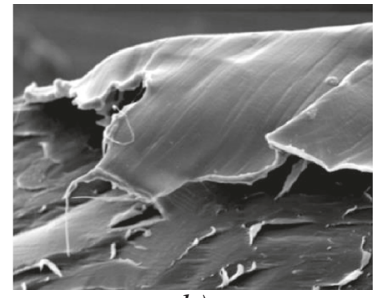

b)

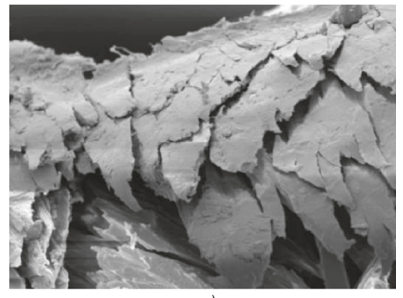

c)

Figure 3: SEM images of tested optical fibres: (a) Longitudinal section (5000x), (b) cross-section near the fracture area (1500x), (c) fracture area $(1000 \times)$ 
microanalysis revealed the presence of fluoride in the thin outer layer, confirming that it corresponds to the polyfluoroalkylmethacrylate cladding of the fibre.

Cross-section images of the fibre are shown in Figures 3-B and 3-C. Figure 3-B depicts a section close to the fracture area and it is shown that the cladding appears as a deformed thin layer and the core as a smoother area, as expected from the observation of the longitudinal section shown in Figure 3-A. The deformation (that can be easily observed in the clad) is a consequence of the stress transmission along the fibre during the test.

The topography in the fracture area is completely different as shown in Figure 3-C: The clad and the core cannot be distinguished anymore, and a single highly deformed area is observed. This observation is in good agreement with the fact that the fibre undergoes a high plastic deformation before reaching the breaking point.

\section{Characterization by differential calorimetry (DSC)}

The thermal transitions of tensile tested and untested POFs are analysed by DSC. According to the data provided by the manufacturer, the glass transition temperature (Tg) of the PMMA core is around $110^{\circ} \mathrm{C}-120^{\circ} \mathrm{C}$. The fluorinated polymers used in this type of POFs usually contain polyfluoroalkylmethacrylate which has a Tg around $75^{\circ} \mathrm{C}$ [12].

Both tensile tested and untested POFs are also scanned on different samples taken near to the mechanical failure region and far from it. In the case of the tested fibres, the scanning provides information of the status after the mechanical test, i.e. it provides relevant information for the interpretation of the mechanical performance.

The results are shown in Figure 4 . In all cases an endothermic transition is detected at $\approx 128^{\circ} \mathrm{C}$, and it is attributed to a melting process, which is confirmed by the presence of an exothermic process in the cooling curve recorded after the dynamic scan (not shown). On the other

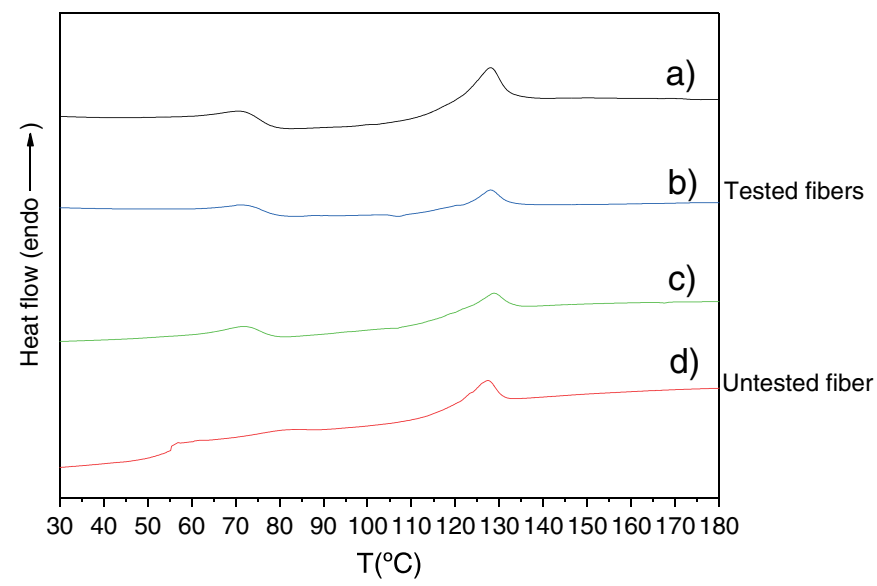

Figure 4: DSC thermograms of specimens recorded at $20^{\circ} \mathrm{C} / \mathrm{min}$. (a) Tested fibre, (b) area close to failure of a tested fibre, (c) area far from failure of a tested fibre, (d) untested fibre hand, the Tg of neat PMMA is not clearly observed, although a shoulder peak at $\sim 120^{\circ} \mathrm{C}$ in the melting transition is intuited. The untested fibres are scanned at different heating rates $\left(2{ }^{\circ} \mathrm{C} / \mathrm{min}, 20^{\circ} \mathrm{C} / \mathrm{min}\right.$ and $40^{\circ} \mathrm{C}$ min) in order to confirm that there are no more overlapping transitions.

An endothermic transition is found at $\approx 71.5^{\circ} \mathrm{C}$ and may be attributed to the glass transition temperature of the cladding polyfluoroalkylmethacrylate material. This transition is clearer in the tested fibres, see Figure 4, curves a), b) and c), than in the untested specimen, see Figure 4D). No differences between the thermal behaviour of specimens taken near and far from the fracture area are observed, and the performance is the same one to the specimens containing random fragments of tested and untested fibre, see Figure 4, curve a).

The thermal transitions measured by DSC showed no differences between the area close to the fracture and far from it, indicating that the orientation of the molecular chains induced by the strain takes place all along the calibration length. Therefore, it can be concluded that deformation occurs in the whole specimen not only at the fracture zone.

\section{Mechanical parameters}

The initial Young's modulus, $\mathrm{E}_{\mathrm{tan}}$, is the slope of the $\sigma-\varepsilon$ curve at the origin in Figure 2. Different tests are made at a strain rate of $0.0005 \mathrm{~s}^{-1}$ and an average Young modulus $\mathrm{E}_{\mathrm{tan}}=3850 \pm 180 \mathrm{MPa}$ is obtained.

To have an estimation of the irreversible variations of the material microstructure, Young's modulus against strain curve is determined by performing tests with several unloading-loading processes. In each of these processes, the slope of the loading (elastic) curve is the material Young's modulus when it has reached the strain corresponding to the start of the unloading. Figure 5 shows the obtained Young's modulus versus strain curve.

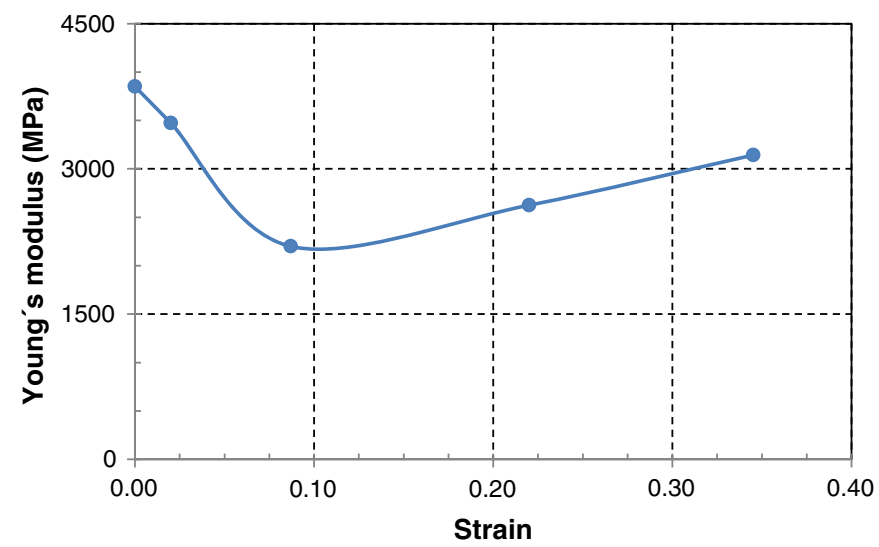

Figure 5: Young's modulus versus strain obtained in a $0.0005 \mathrm{~s}^{-1}$ strain rate test 
The rapid initial reduction of $\mathrm{E}$ is associated with the breaking of the bonds of the molecular chains and their relative displacements until new stable positions. A minimum value of $\mathrm{E}$ is reached at $\varepsilon \approx 0.10$. From this point the material stiffness starts to increase until the molecular chains reach their maximum elongation when the chains begin to stretch. The point of inflection in the $\sigma-\varepsilon$ curve (at $\varepsilon \approx 0.10$ ) corresponds roughly to the point of minimum Young's Modulus in the E- $\varepsilon$ curve

Two procedures are used to estimate the yield stress, $\sigma_{\mathrm{y}}$. In the first approach, $\sigma_{\mathrm{y}}$ is estimated as the first relative maximum in the $\sigma-\varepsilon$ curve, which leads to a value of $\sigma_{y}=78 \pm 3 \mathrm{MPa}$ for $0.0005 \mathrm{~s}^{-1}$ strain rate tests. The second estimation procedure approximates $\sigma_{\mathrm{y}}$ as the intersection point of two straight lines: the tangent line to the $\sigma-\varepsilon$ curve at the origin and the straight line that best fits to the straight segment at the beginning of the softening zone. This second procedure leads to $\sigma_{\mathrm{y}}=80 \pm 3 \mathrm{MPa}$ at the same strain rate.

The elastic $\varepsilon^{\mathrm{e}}$ and plastic $\varepsilon^{\mathrm{p}}$ strain components are determined using both Equation (2) and assuming a polynomial fit of $E$ from Figure 5. Their time variations are depicted in Figure 6.

After the yield stress is reached, the elastic strain component is practically stabilized at $\varepsilon \approx 0.02$, see Figure 6A. This effect suggests that the intermolecular forces no longer have the capacity of returning the chains to their original relative positions. Nevertheless, the plastic strain component dramatically increases, as shown in Figure 6-B. This fact suggests the progressive increase in the number of chains displaced towards stable positions.

The tensile strength is determined by applying the Considère criterion [13], given by:

$\frac{d \sigma}{d \varepsilon}=\sigma$

to the measurements shown in Figure 2, which yields $\sigma=85+/-4 \mathrm{MPa}$ (at $\varepsilon=0.32$ ). This value reveals that the tensile strength and ultimate stress reach very close values. The mean value of the ultimate strain (indirect measure of ductility of the material) is established as $\varepsilon_{R}=0.35$.

\section{Influence of strain rate in the mechanical parameters}

A wide set of tests are made at different load velocities to analyse the influence of the strain rate, within the range of velocities in the quasi-static regime. Table 1 includes the measured values of Young's modulus, yield stress, tensile strength and ultimate strain.

As the strain rate increases, all the yield stress, the tensile strength, the ultimate strain and Young's modulus increase; although E increases at a smaller extent. This behaviour is in agreement with previous works [6].

\section{Thermo-mechanical behaviour}

The heating process because of elastic and plastic strain can be incorporated into the thermal balance equation as heat source terms. For a material with elastic-plastic behaviour, the energy-balance equation which provides the temperature variation under axial stress is defined as Equation (4) $[7,14]$

$\dot{T}-\lambda \Delta T=\frac{\alpha}{\rho C_{p}} E T_{o} \dot{\varepsilon}^{e}+\frac{\beta}{\rho C_{p}} \sigma \dot{\varepsilon}^{p}$

being $T_{O}$ the absolute temperature, $\dot{\varepsilon}^{e}$ and $\dot{\varepsilon}^{p}$ the elastic and plastic components of the strain rate (assuming additive

Table I: Mechanical parameters versus strain rate

\begin{tabular}{llll}
\hline Strain rate & $\dot{\varepsilon}=0,0005 \mathrm{~s}^{-1}$ & $\dot{\varepsilon}=0,0025 \mathrm{~s}^{-1}$ & $\dot{\varepsilon}=0,0072 \mathrm{~s}^{-1}$ \\
\hline $\begin{array}{l}\text { Initial Young's } \\
\text { modulus (MPa) }\end{array}$ & 3850 & 3870 & 3930 \\
$\begin{array}{l}\text { Yield stress } \\
(\mathrm{MPa})\end{array}$ & $78 / 80$ & $85-87$ & $105-108$ \\
$\begin{array}{l}\text { Tensile strength } \\
(\mathrm{MPa})\end{array}$ & 85 & 120 & 150 \\
Ultimate strain & 0.35 & 0.38 & 0.42 \\
\hline
\end{tabular}
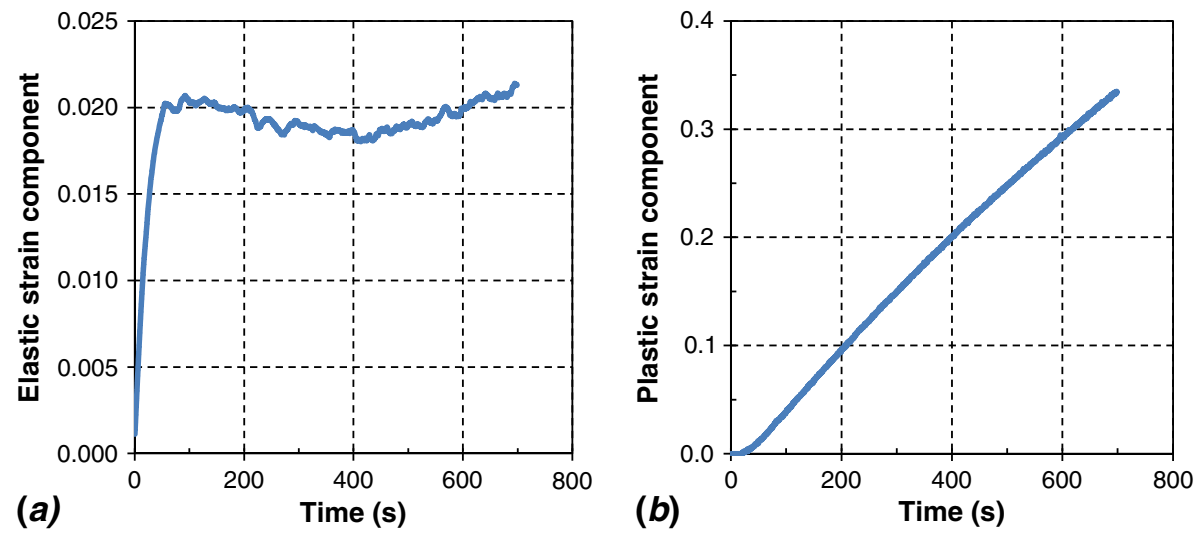

Figure 6: Strain components versus time, strain rate of $0.0005 \mathrm{~s}^{-1}$ : (a) elastic $\varepsilon^{\mathrm{e}}$, (b) plastic $\varepsilon^{\mathrm{p}}$ 
decomposition of the strain), $\Delta \mathrm{T}$ the temperature increase and $\sigma$ the stress. Material parameters defined as $\lambda, \alpha, \rho, C_{P}$ and $\mathrm{E}$ are the thermal diffusivity, the thermal expansion coefficient, density, the specific heat at constant pressure and Young's modulus, respectively. $\beta$ is the Quinney-Taylor coefficient which defines the plastic work fraction converted into heat. $\mathrm{T}_{0}$ is the initial temperature.

In Equation (4), the term $\frac{\alpha}{\rho C_{p}} E T_{o} \dot{\varepsilon}^{e}$ represents the temperature variation associated with the elastic deformation (reversible) and whose sign is dependent on the sign of $\dot{\varepsilon}^{e}$, whereas the term $\frac{\beta}{\rho C_{p}} \sigma \dot{\varepsilon}^{p}$ represents the temperature variation associated with the plastic deformation (irreversible) and whose sign is always positive.

Equation (4) is shown here just to emphasize that under monotonic tensile loading, i.e. a situation where elastic and plastic strains are both increasing, the two source terms have opposite sign leading to the temperature decrease and increase by the elastic and plastic terms, respectively.

Using the IRC, the temperature field of the calibration length of the fibre during its deformation process was obtained. From de camera records, the time evolution of maximum temperature, $T_{\max }$, was determined in two specimen zones: the central third and a lateral third, where, afterwards, the breakage occurred.

Figure 7-A depicts the $\sigma-\varepsilon$ curve obtained in a test at a load velocity of $60 \mathrm{~mm} / \mathrm{min}$ corresponding to $\dot{\varepsilon}=0.0072 \mathrm{~s}^{-1}$. In this test, the specimen breakage occurred at a value of $\varepsilon=0.42$, being the elastic and plastic strain components $\varepsilon^{\mathrm{e}}=0.04$ and $\varepsilon^{\mathrm{p}}=0.38$, respectively, see Figure $7-\mathrm{B}$, recalling Equation (2). $\mathrm{T}_{\max }$ time evolution in the selected specimen sections is shown in Figure 7-C.

At the beginning of the test, in the strain interval [0.0 0.04] (see Figure 7-C), the specimen surface temperature decays uniformly $\left(\Delta \mathrm{T} \approx-0.5^{\circ} \mathrm{C}\right)$ from the initial temperature $\left(\mathrm{T}=19.8^{\circ} \mathrm{C}\right)$. This is because the elastic strain component is larger than the plastic strain counterpart. The stress value of $105 \mathrm{MPa}$ at $\varepsilon=0.04$ can be defined as the material yield stress at $\dot{\varepsilon}=0.0072 s^{-1}$. From $\varepsilon=0.04$ the specimen surface temperature rises progressively from its minimum value $\mathrm{T}=19.2^{\circ} \mathrm{C}$ to $\mathrm{T}=22.2^{\circ} \mathrm{C}$ in both the central third and the lateral third of the specimen. This temperature increase is because of the progressive increase of the plastic strain component which reaches values far larger than those of the elastic strain component (see Figure 7-B).
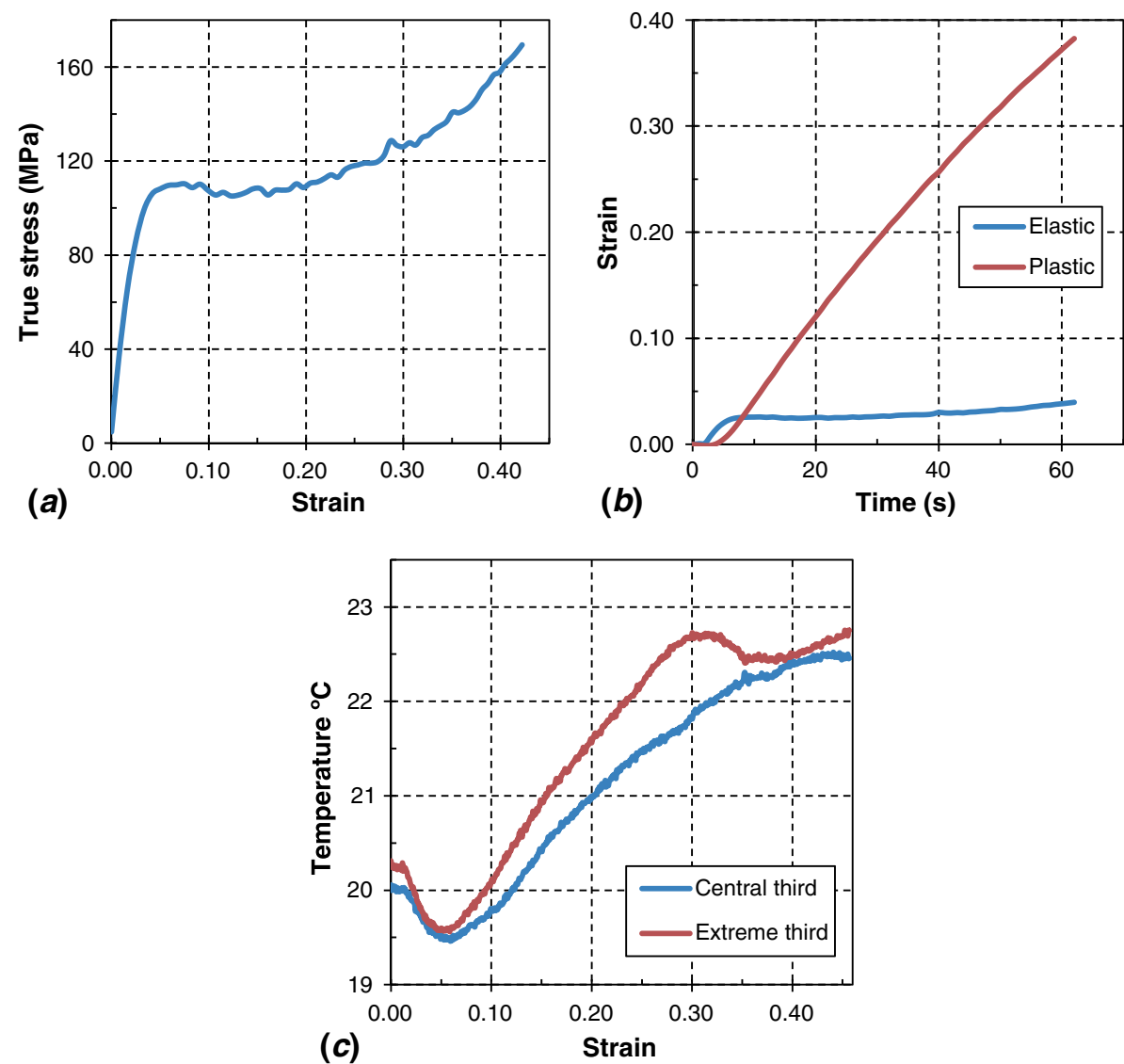

Figure 7: Measurements recorded in a test at $\dot{\varepsilon}=0,0072 \mathrm{~s}^{-1}$. (a) True stress versus true strain, (b) elastic and plastic components versus time, (c) maximum temperature of the specimen surface in the central and in the lateral thirds versus strain 
A subtle softening can be seen after $\varepsilon=0.06$. This is a standard feature of stress-strain curves of many polymers. In the present case it is not related with a thermal softening effect because of the relatively small measured value of $\Delta \mathrm{T} \approx 3{ }^{\circ} \mathrm{C}$.

For $\varepsilon \approx 0.10$, both the $\sigma-\varepsilon$ curve (see Figure $7-A$ ) and the $\mathrm{T}_{\max }-\varepsilon$ curve (see Figure $7-\mathrm{C}$ ) show an inflexion point. For $\varepsilon>0.10$, both the increase of the $\sigma-\varepsilon$ curve slope and the decrease of $\mathrm{T}_{\max }-\varepsilon$ curve slope, respectively, suggest the progressive stretching of the molecular chains and their consequent elastic deformation.

In the lateral third of the specimen, the surface temperature increase is quicker, since the fracture is going to happen in this zone. Here, also the plastic deformations as well as the density of the molecular chains that are straightened are larger than in the central third. It is also observed that the temperature reaches a maximum at $\varepsilon=0.3$. This is because the molecular chains in the fracture zone are mainly stretched and, therefore, deformed elastically, which implies a temperature decrease.

\section{Photo-mechanical behaviour}

Figure 8 depicts the normalized received optical power versus strain measured during tensile tests for strain rates of $0.0004 \mathrm{~s}^{-1}, 0.0028 \mathrm{~s}^{-1}$ and $0.0084 \mathrm{~s}^{-1}$, respectively. Each curve represents the averaging of three trials under the same test conditions.

From Figure 8 it is observed that the received optical power diminishes up to $15-20 \%$ while deformation increases. Within the elastic deformation range $(\varepsilon<0.04)$, the decrease in relative optical power is smaller than $2 \%$. Figure 8 also reflects a slight dependency of the optical power loss with the strain rate. For the same deformation,

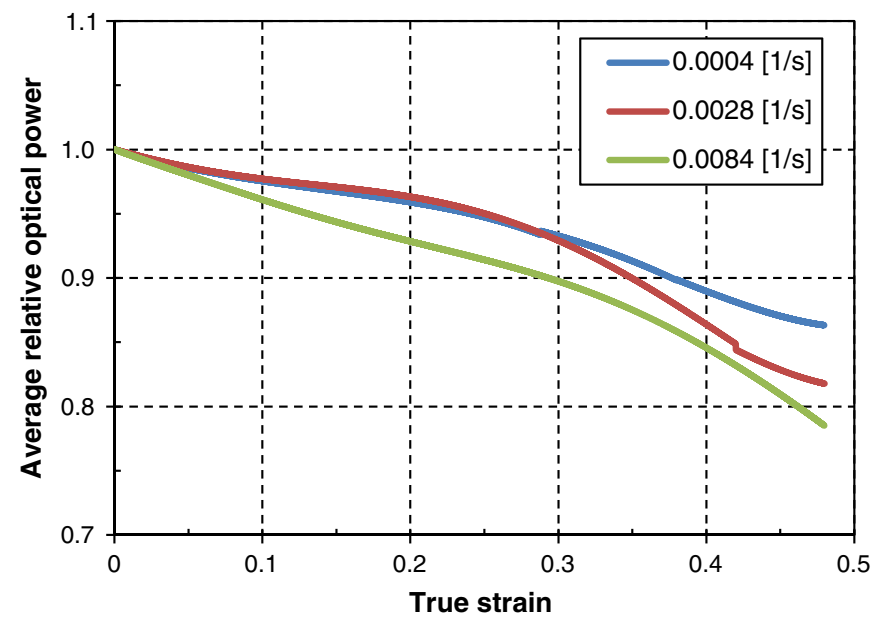

Figure 8: Normalized received optical power versus strain in tensile tests at strain rates of $0.0004 \mathrm{~s}^{-1}, 0.0028 \mathrm{~s}^{-1}$ and $0.0084 \mathrm{~s}^{-1}$ as $\dot{\varepsilon}$ is increased (in the range of a quasi-static strain rate) the received optical power decreases.

It is well-known that temperature affects the refractive index (RI) of polymers. In Ref. [18], different curves describing the RI versus temperature dependency are provided for the PMMA material. From those figures, it is seen that a $\Delta \mathrm{T}=2.5^{\circ} \mathrm{C}$ starting from $\mathrm{T}=20^{\circ} \mathrm{C}$ results in a 0.0003 RI decrease. Although the RI of the fibre core is a fundamental parameter to describe light propagation inside optical fibres, such a measured RI variation because of temperature changes cannot be considered as the cause of the optical power loss showed in Figure 8.

Because of its polymeric nature the fibre core has a viscoelastic behaviour. Creep and relaxation are the phenomenological manifestations of the viscoelastic behaviour. To analyse the influence of the viscoelastic nature of the fibre core in the optical power loss, a relaxation test with simultaneous measurement of the optical power is performed. The fibre is elongated at a rate of $3 \mathrm{~mm} / \mathrm{min}$ up to $\Delta \mathrm{L}_{0}=13.5 \mathrm{~mm} \quad\left(\varepsilon_{0}=0.13\right)$ which corresponds to an applied force $F_{0}=54 \mathrm{~N}\left(\sigma_{0}=80 \mathrm{MPa}\right)$. From this point, the fibre length is kept constant during 6500 s, and the tension sustained by the fibre is constantly monitored.

Figure 9-A shows the true stress time evolution starting at $\varepsilon_{0}=0.13$. From this figure it can be concluded that from the stress applied when deformation $\varepsilon_{0}=0.13$ is reached, the material relaxes (i.e. the stress diminishes) as expected.

Figure 9-B shows the time evolution of the received optical power in the test at a constant strain. It is seen that even with a constant deformation in the viscoelastic relaxation test, the received optical power decreases. This fact suggests that the optical power loss in the fibre depends not only on the strain level but also on its stress state. It must be considered that both the true stress and the true strain (which remains constant in the relaxation test) are macroscopic variables. Because the applied stress must, however, be equilibrated by a system of intermolecular forces it can be concluded that any change in stress that occurs at a constant strain is a manifestation of the changes in the microstructure of the material. These changes, in turn, would explain the decrease of optical power during the relaxation test. The polymeric coatings of glass optical fibres are usually viscoelastic materials. The viscoelastic behaviour of polymeric coatings is analysed in [16] to understand the long term fibre stresses and the related microbending loss and refractive index changes. In POF fibres this effect can be even more relevant. The refractive index change in long period Bragg gratings sensors based on microestructured POF viscoelastic properties is also reported in [17], with appreciable wavelength shifts at a constant strain of $4 \%$. The microbending loss and refractive index changes can be the responsible for optical power decrement under a high constant strain. 

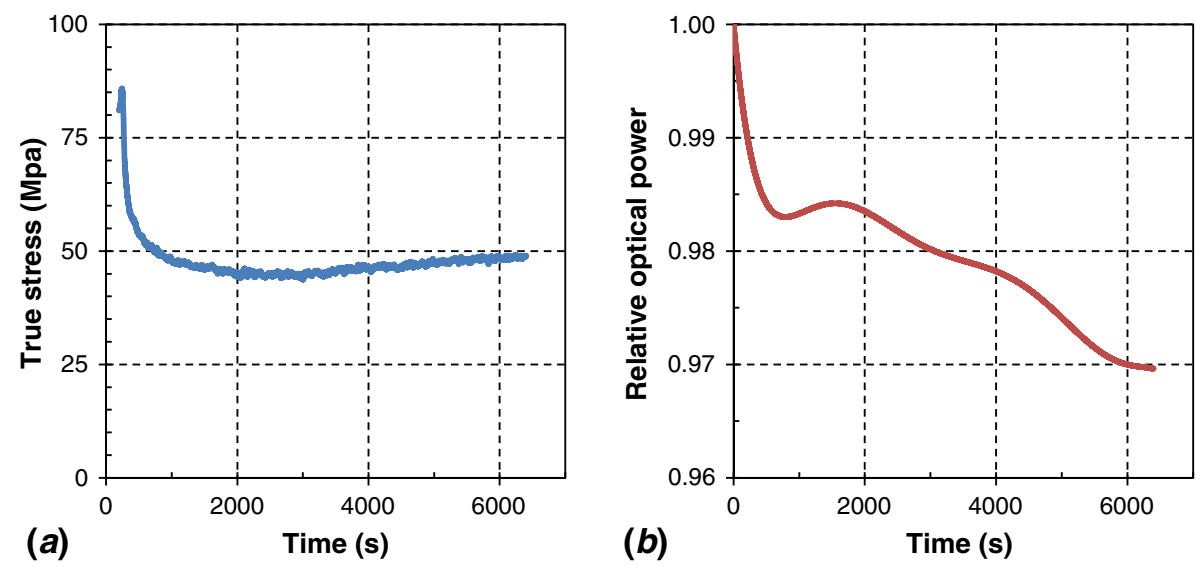

Figure 9: (a) True stress time evolution, (b) average relative optical power time evolution in a relaxation test

POF has already been embedded in composite materials to monitor damage development. The non-linearity observed in the optical response [18] was an indication of local deformation of POF resulting from the damage in the host and hence demonstrated the viability of the technique for detection of transverse damage in composite materials. Some surface mounting POF sensors were also developed $[19,20]$.

Nevertheless, depending on the metric, either wavelength shifts or optical power variations, relaxation tests should always be performed prior to determine the sensor application scenario. In the case of intensity based sensors, specific self-reference techniques should be included to record the sensor history. So intensity-based POF sensors could offer a simple, not expensive yet effective approach in monitoring specific aspects in SHM applications, in particular when high-resolution and precise measurements are not key requirements in the application.

Anyway, further research is needed in order to determine the full POF characteristics under mechanical stress and the strain limits where those sensors can be used in SHM to correctly interpret the POF sensor response.

\section{Conclusions}

Tensile tests at different strain rates in quasi-static conditions are performed in step index polymer optical fibres with poly(methyl methacrylate) core (PMMA). Young's modulus, yield stress and tensile strength are derived from experimental results. Young's modulus evolution is determined by tests with unloading-loading processes. Simultaneous measurements of the evolution of specimen surface temperature and the received optical power are also performed.

From the experimental results, it is found that Young's modulus, the yield stress, the tensile strength and optical power loss increase when the strain rate increases in the quasi-static range.

On the other hand, the evolution of both the elastic and plastic components of the strain is analysed together with the evolution of the specimen surface temperature; this correlation is found to be qualitatively in agreement with the theoretical models. A temperature decrease of $0.4^{\circ} \mathrm{C}$ under elastic strain, up to $\varepsilon=0.04$, and an increase of $3{ }^{\circ} \mathrm{C}$ mainly under plastic strain, up to $\varepsilon=0.45$, are observed in tests at $\dot{\varepsilon}=0,0072 s^{-1}$. This effect can be of interest when analysing cross-sensitivity considerations on simultaneous measurement of temperature and strain using fibre Bragg grating (FBG) POF sensors.

The morphological characterization of the optical fibre under stress using scanning electron microscope images and differential calorimetry techniques reveals an endothermic transition attributed to the disorganization during the POF traction test.

Finally, an empiric correlation between the microstructural deformation process (relative displacements of the direction of the applied load) and the evolution of the Young's modulus, the temperature in the specimen surface and the optical power loss are also addressed. Depending on the application, a time-dependent effect such as relaxation during constant strain should be performed to delimit POF sensor resolution.

\section{ACKNOWLEDGEMENTS}

This work was supported by the Spanish Ministry of Economía y Competitividad under grants CCG06-UC3M/DPI-0796 and TEC2012-37983-C03-02 and Comunidad de Madrid under grant S2013/MIT-2790 (SINFOTON-CM). The support of J. C. Torres in the optical power measurements is gratefully acknowledged. 


\section{REFERENCES}

1. Kuang, K. S. C., Quek, S. T., Koh, C. G., Cantwell, W. J. and Scully, P. J. (2009) Plastic optical fibre sensors for structural health monitoring: a review of recent progress. Journal of Sensors. Hindawi Publishing Corporation, 2009, DOI: 10.1155/2009/312053.

2. Tapetado, A., Montero, D. S., Webb, D. J., Vázquez, C. (2014) Self-referenced optical intensity sensor network using POFBGS for biomedical applications. Sensors. 14, 24029-24045.

3. Tapetado, A., Pinzon, Castillo, P. J., Zubía J., Vázquez C. (2015) Polymer optical fiber temperature sensor with dual-wavelength compensation of power fluctuations. IEEE J. Lightwave Technol. 33, 2716-2723.

4. Xiong, Z., Peng, G. D., Wu, B. and Chu, P. L. (1999) Highly tunable Bragg gratings in single-mode polymer optical fibres. Photon. Technol. Lett., IEEE. 11, 352-354.

5. Kuang, K. S. C., Cantwell, W. J. and Scully, P.J. (2002) An evaluation of a novel plastic optical fibre sensor for axial strain and bend measurements. Meas. Sci. Technol. 13, 1523-1534.

6. Kiesel, S., K. Peters, Hassan, T. and Kowalsky, M. (2007) Behavior of intrinsic polymer optical fiber sensor for largestrain applications. Measurement Science and Technology. 18, 3144-3154.

7. Taylor, G. I. y. , Quinney, H. (1933) Latent energy remaining in a metal after cold working. Proc. Royal Soc. 143, 307-326.

8. Pérez-Castellanos J.L., Guzmán, R., Rusinek A. and Meléndez J. (2010) Temperature increment during quasi-static compressive tests using Mg metallic alloys. Mater. Design. 31, 3259-3269.

9. Hallman, J.R. (1971) Ignition characteristics of plastics and rubber, Dissertation at the University of Oklahoma, Norman, Oklahoma.

10. Jiang C., Kuzyk M.G., Ding J.L., Johns W.E. and Welker D. (2002) Fabrication and mechanical behaviour of dye-doped polymer optical fibre. J. Appl. Phys. 92, 4-12
11. Ward, I.M. (1983) Mechanical Properties of Solid Polymers, John Wiley \& Sons, Chichester.

12. Emslie, C. (1988) Review polymer optical fibers. J. Mat. Sci. 23, 2281-2293.

13. Considère A. (1885) Ann. Ponts Chaussées 9 574-575. [31] W.A. Backofen,

14. Farren, W.S., Taylor, G.I. (1925) The heat developed during plastic extension of metals. Proc. Roy. Soc. London. A107, 422-451.

15. Kasarova S.N., Sultanova N.G. and Nikolov I.D. (2010) Temperature dependence of refractive characteristics of optical plastic. J. Phys: Conf. Series. 253, 012028. IOP Publishing Ltd.

16. Yu-Ching, Yang, Shu-Huang, Sun, Shao-Shu, Chu, Jung-Chang, Hsu (2004) Analysis of long-term optical effects in doublecoated optical fibers induced by hydrostatic pressure and thermal loading. Optic. Quantum Electron. 36, 597-613

17. Maryanne C J Large, Joshua Moran and Lin Ye, (2009) The role of viscoelastic properties in strain testing using microstructured polymer optical fibres (mPOF). Meas. Sci. Technol. 20, 034014 (6pp)

18. Takeda N. (2002) Characterization of microscopic damage in composite laminates and real-time monitoring by embedded optical fiber sensors. Int. J. Fatigue. 24, 281-289.

19. Durana G., Wong, Y. M., Scully, P. J., Bartlett, R. J., Kuang, K. S. C. and Cantwell, W. J. (2009) Use of a novel fiber optical strain sensor for monitoring the vertical deflection of an aircraft flap. IEEE Sensors J. 9, 1219-1225.

20. Wong Y. M., Scully P. J., Bartlett R. J., Kuang K. S. C. and Cantwell W. J. (2003) Plastic optical fibre sensors for environmental monitoring: biofouling and strain applications. Strain. 39, 115-119. 